\title{
Correlation-induced spin freezing transition in FeSe: A dynamical mean field study
}

\author{
Ansgar Liebsch ${ }^{1}$ and Hiroshi Ishida ${ }^{2}$ \\ ${ }^{1}$ Institut für Festkörperforschung, Forschungszentrum Jülich, 52425 Jülich, Germany \\ ${ }^{2}$ College of Humanities and Sciences, Nihon University, Tokyo 156, Japan
}

(Received 10 September 2010; published 5 October 2010)

\begin{abstract}
The effect of local Coulomb interactions on the electronic properties of FeSe is explored within dynamical mean field theory combined with finite-temperature exact diagonalization. The low-energy scattering rate is shown to exhibit non-Fermi-liquid behavior caused by the formation of local moments. Fermi-liquid properties are restored at large electron doping. In contrast, FeAsLaO is shown to be located on the Fermi-liquid side of this spin freezing transition.
\end{abstract}

DOI: $10.1103 /$ PhysRevB.82.155106

PACS number(s): 71.20.Be

\section{INTRODUCTION}

The recent discovery of high-temperature superconductivity in iron-based pnictides ${ }^{1}$ and chalcogenides ${ }^{2}$ has led to an intense discussion concerning the role of Coulomb correlations in these materials. Although compounds such as FeAsLaO (1111), $\mathrm{BaFe}_{2} \mathrm{As}_{2}$ (122), $\mathrm{LiFeAs}$ (111), and FeSe (11) all have rather similar one-electron properties, a variety of experiments suggest significant differences. For instance, photoemission measurements show that $\mathrm{FeAsLaO}$ is moderately correlated, with about $50 \% 3 d$ band narrowing and effective-mass enhancement of about $2-3 .^{3}$ In contrast, several photoemission data on $\mathrm{FeSe}_{x} \mathrm{Te}_{1-x}$ samples reveal larger effective-mass enhancement and stronger band narrowing, ${ }^{4-8}$ optical measurements exhibit incoherent spectral features indicative of a pseudogap, ${ }^{9}$ and several transport measurements show large deviations from Fermi-liquid (FL) behavior. ${ }^{10-13}$ Moreover, recent theoretical work ${ }^{14}$ suggests that Coulomb interactions in (11) compounds ought to be less well screened than in (1111) systems.

The aim of this paper is to elucidate the origin of the experimentally observed bad-metallic behavior of FeSe. Using an accurate single-particle description of the electronic structure together with appropriate interaction parameters, ${ }^{14}$ and evaluating the influence of local Coulomb interactions within dynamical mean field theory (DMFT), ${ }^{15}$ we show that correlations are strong enough to give rise to the formation of $\mathrm{Fe} 3 d$ local moments, implying non-Fermi-liquid behavior, where electronic states at the $E_{F}$ exhibit a finite lifetime. Moreover, we demonstrate that these properties are caused by a nearby doping-driven spin freezing transition, i.e., Fermi-liquid behavior is restored toward larger electron doping whereas hole doping reinforces bad-metallic properties. Using the same approach for $\mathrm{FeAsLaO}$ we show that Coulomb interactions are too weak to cause spin freezing so that this system merely exhibits moderate effective-mass enhancement. ${ }^{16}$

Thus, FeSe appears to be a material which exhibits bad metallicity induced by a spin freezing transition, a mechanism recently identified by Werner et al. ${ }^{17}$ in a three-band model. For FeAsLaO, Haule et al. ${ }^{18}$ showed that large Coulomb interactions lead to the formation of local moments and large scattering rates while Ishida and Liebsch ${ }^{19}$ discussed the spin freezing transition as a function of Coulomb inter- action and doping. Bad-metallic behavior in FeSe was also found in DMFT studies by Craco et al. ${ }^{20}$ and Aichhorn et $a l .{ }^{21}$ The spin freezing origin of this behavior, however, was not investigated in these works. Hansmann et al. ${ }^{22}$ studied Coulomb correlations in FeAsLaO within a four-band model and discussed the striking difference between the large static magnetic moment and the smaller dynamically screened moment.

\section{THEORY}

To account for local Coulomb interactions among Fe $3 d$ electrons we use exact diagonalization (ED) (Ref. 23) which we have recently extended to five orbitals. ${ }^{19}$ Discretization of the lattice surrounding the local impurity is achieved by using ten bath levels, which yields excellent projections of the lattice Green's function onto the cluster consisting of impurity plus bath. Because of the very large size of the Hilbert space of this 15 level system (the largest spin sector has dimension $\sim 40 \times 10^{6}$ ) the spacing of excited states is very small so that finite-size errors are greatly reduced. Moreover, ED has the advantage of allowing for rotationally invariant Hund exchange. This is of crucial importance for the spin freezing transition since omission of spin-flip and pairexchange interactions leads to a significant shift of the Fermi-liquid to non-FL (NFL) phase boundary. For computational reasons we restrict ourselves to the lowest excited states, which are relevant near the $T \rightarrow 0$ limit. Only the paramagnetic phase is considered. Further calculational details can be found in Ref. 19.

The electronic properties of FeSe are formulated in terms of the effective low-energy model recently derived by Miyake et al. ${ }^{14}$ In this scheme, standard band-structure calculations within the local-density approximation were carried out, analogous to previous work in Refs. 24 and 25 From these results, maximally localized Wannier functions were constructed for the $\mathrm{Fe} 3 d$ bands, following the procedure discussed in Ref. 26. The intersite transfer integrals are then derived from the matrix elements of the Kohn-Sham Hamiltonian $H(\mathbf{k})$ in the basis of these Wannier functions. Finally, the constrained random-phase approximation (RPA) developed by Aryasetiawan et al. ${ }^{27}$ was used to determine the effective Coulomb and exchange interaction parameters, which account for screening via Se $4 p$ orbitals. Because of 

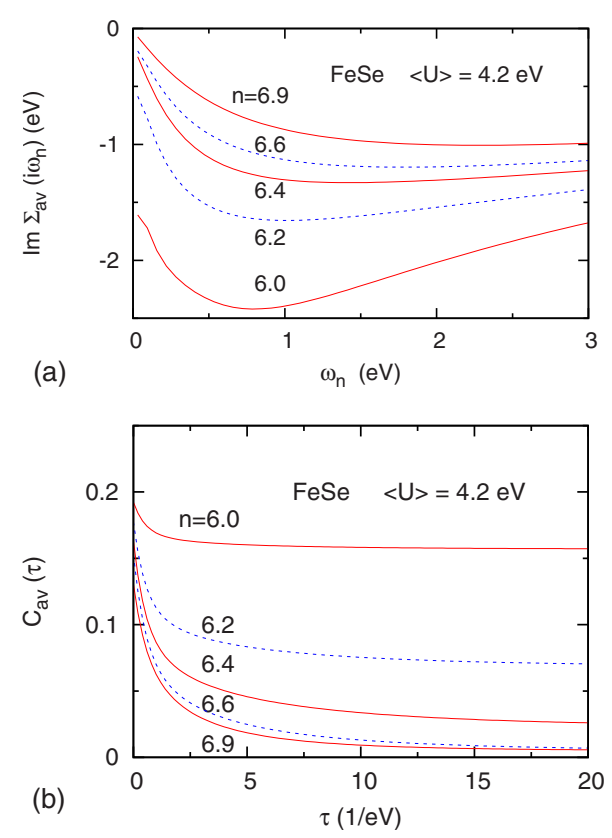

FIG. 1. (Color online) Orbital average of (a) imaginary part of $3 d$ self-energy and (b) spin-spin correlation function for FeSe at various occupancies. For $n>6.3, \ldots, 6.5$, the self-energy exhibits FL behavior and the spin susceptibility is Pauli type. At smaller occupancy, the self-energy reveals NFL behavior and the susceptibility becomes Curie-Weiss type, giving rise to bad metallicity caused by spin freezing $(T=0.01 \mathrm{eV})$.

the planar structure of FeSe, the interaction parameters exhibit appreciable orbital dependence. According to these results, Coulomb interactions in FeSe are typically 50\% larger than in FeAsLaO. This follows from the larger $z$ spacing and concomitant smaller spread of Wannier orbitals, and from the reduced number of screening channels in FeSe. The oneelectron transfer integrals $t_{m n}(\mathbf{R})$ and interaction matrices $U_{m n}$ and $J_{m n}$ for FeSe are given in Tables VII and VIII of Ref. 14, respectively. The average intraorbital Coulomb interaction is $\langle U\rangle=4.2 \mathrm{eV}$ and the average Hund exchange is $\langle J\rangle=0.5 \mathrm{eV}$.

\section{RESULTS AND DISCUSSION}

Figure 1(a) shows the orbital average of the imaginary part of the $3 d$ self-energy components, $\operatorname{Im} \Sigma_{m}\left(i \omega_{n}\right)$, for various $3 d$ occupancies ( $\omega_{n}$ are Matsubara frequencies). The one-electron transfer integrals and interaction matrix elements are kept fixed at the values for $n=6$. Fermi-liquid behavior, with $\operatorname{Im} \Sigma_{m}\left(i \omega_{n}\right) \sim \omega_{n}$ in the limit of small $\omega_{n}$, combined with effective-mass enhancement $m^{*} \approx 3$, is found for $n>6.3, \ldots, 6.5$. At lower $3 d$ occupancy, however, the imaginary part of the self-energy exhibits a finite onset, implying bad-metallic behavior due to a finite lifetime of electronic states at the Fermi level $E_{F}$. Even stronger correlationinduced scattering occurs on the hole doping side for $n<6$ (see below).

The corresponding orbital components of the low-energy limit of the $3 d$ self-energy, $\gamma_{m}=-\operatorname{Im} \Sigma_{m}\left(i \omega_{n} \rightarrow 0\right)$, are shown in Fig. 2(a) as a function of Fe $3 d$ occupancy. Small scatter-
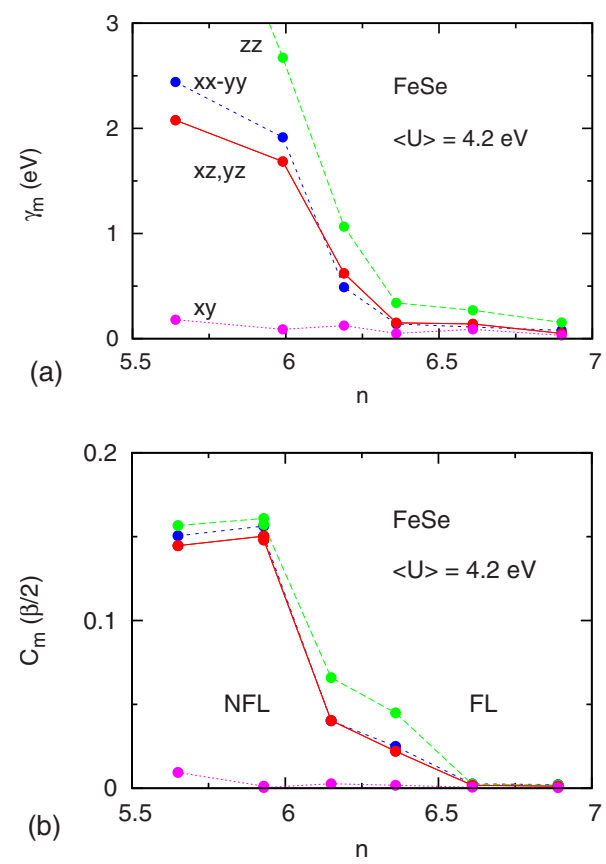

FIG. 2. (Color online) (a) Low-energy scattering rates $\gamma_{m}$ $=-\operatorname{Im} \Sigma_{m}\left(i \omega_{n} \rightarrow 0\right)$ and (b) long-time limit of orbital-dependent spin-spin correlations $C_{m}(\tau=\beta / 2)$ of FeSe as functions of $3 d$ occupancy. Near $n=6$, the bad-metallic behavior of $\gamma_{m}>0$ is caused by the formation of local moments. Increasing electron doping restores Fermi-liquid properties whereas hole doping enhances bad-metallic properties.

ing rates consistent with Fermi-liquid behavior are found for $n>6.5$ but increasing scattering rates are obtained at lower electron doping, except for the $d_{x y}$ orbital. This orbital appears to be less correlated since the width of its singleparticle density of states is about $0.5 \mathrm{eV}$ wider than that of the other $3 d$ components. ${ }^{14}$ On the hole doping side, the scattering rates continue to increase, indicating the proximity of the paramagnetic Mott insulating phase in the limit of half filling at $n=5$.

To identify the origin of this change from Fermi-liquid to non Fermi-liquid behavior we have evaluated the spin-spincorrelation functions $C_{m}(\tau)=\left\langle S_{m z}(\tau) S_{m z}(0)\right\rangle$, where $\tau$ denotes imaginary time. In the Fermi-liquid regime, these functions decay with $\tau$ so that $C_{m}(\tau=\beta / 2)$ is very small $(\beta=1 / T)$. The susceptibilities $\chi_{m} \sim \int_{0}^{\beta} d \tau\left\langle S_{m z}(\tau) S_{m z}(0)\right\rangle$ then are Pauli type, i.e., independent of temperature. Figure 1(b) shows the orbital average $C_{a v}(\tau)=\frac{1}{m} \sum_{m} C_{m}(\tau)$ as a function of $\tau$. For $n$ $>6.3, \ldots, 6.5, C_{a v}(\tau)$ decays with $\tau$, confirming the Fermiliquid behavior of the self-energy. At lower $3 d$ occupancy, however, $C_{a v}(\tau)$ reaches increasingly larger constant values near $\tau=\beta / 2$, as illustrated in Fig. 2(b). The corresponding susceptibilities $\chi_{m}$ then become proportional to $1 / T$, as expected for Curie-Weiss behavior associated with the formation of local moments.

A similar spin freezing transition was recently found by Werner et al. ${ }^{17}$ for a degenerate three-band model near $n=2$. Using continuous-time quantum Monte Carlo DMFT, the paramagnetic phase at moderate $U$ was shown to exhibit Fermi-liquid properties at small $n$. For $n>1.5$, an incoherent metallic phase appears, where the imaginary part of the self- 


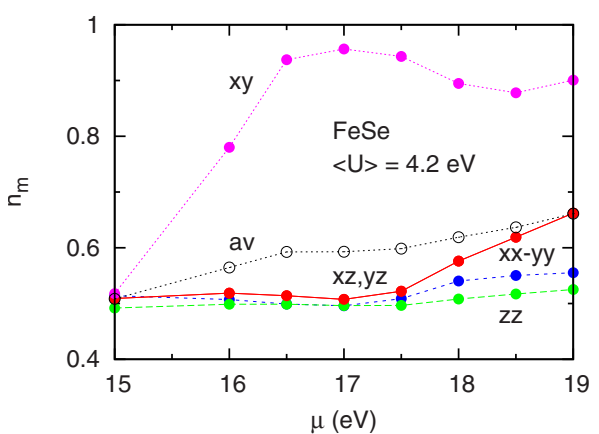

FIG. 3. (Color online) Fe $3 d$ orbital occupancies as functions of chemical potential. $n_{a v}$ denotes the average occupancy. Nominal occupancy $n=6$ is reached near $\mu=17 \mathrm{eV}$ while at $\mu=15 \mathrm{eV}$ all bands are approximately half filled.

energy exhibits a finite onset at $\omega=0$ due to the formation of local moments.

An important consequence of Coulomb correlations is the redistribution of charge among orbitals. Figure 3 shows the $3 d$ orbital occupancies as a function of doping. At $n=6$, these values differ significantly from their uncorrelated values: $n_{x z, y z, x^{2}-y^{2}, x y, z^{2}}=(0.54,0.54,0.56,0.60,0.76), \quad$ indicating strong interorbital charge transfer induced by Coulomb interactions. In particular, $n_{x y}$ reaches nearly unity while the other $n_{m}$ are close to half filling. Despite this orbital polarization, the spectral distributions (not shown here) reveal considerable intensity near $E_{F}$, suggesting that the system is not in a Mott phase, where one of the subbands is filled and the others are split into lower and upper Hubbard bands. The $3 d$ self-energies and spin-correlation functions also exhibit orbital dependence. Typically, the $x y$ component is less correlated because of the larger single-particle width of its density of states. ${ }^{14}$

To investigate the relationship between orbital polarization and local-moment formation, we have studied Coulomb correlations in a fully degenerate five-band model, with bandwidth and Coulomb interactions of similar magnitude as in FeSe. As shown in Fig. 4, this system exhibits the same kind of spin freezing transition as discussed above for FeSe.

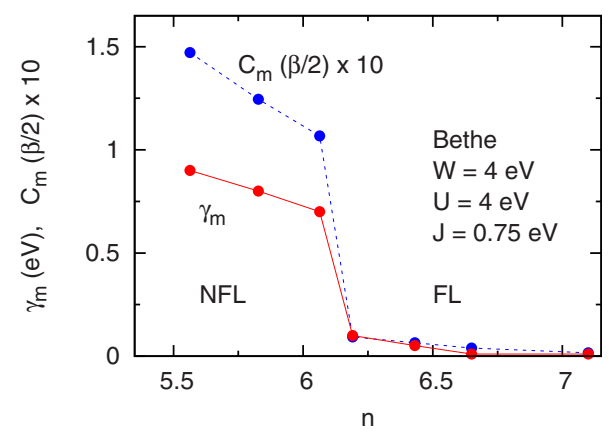

FIG. 4. (Color online) Low-energy scattering rates and spin-spin correlations derived within ED/DMFT for a degenerate five-band model with Bethe lattice density of states with $W=4 \mathrm{eV}$. For $U$ $=4 \mathrm{eV}, J=0.75 \mathrm{eV}$, the spin freezing transition occurs at $n=6.2$ with Fermi-liquid behavior at $n>6.2$ and increasing bad metallicity for $n<6.2$. For $U=4 \mathrm{eV}, J=0.5 \mathrm{eV}$ (not shown), the transition occurs at $n=6.1$.
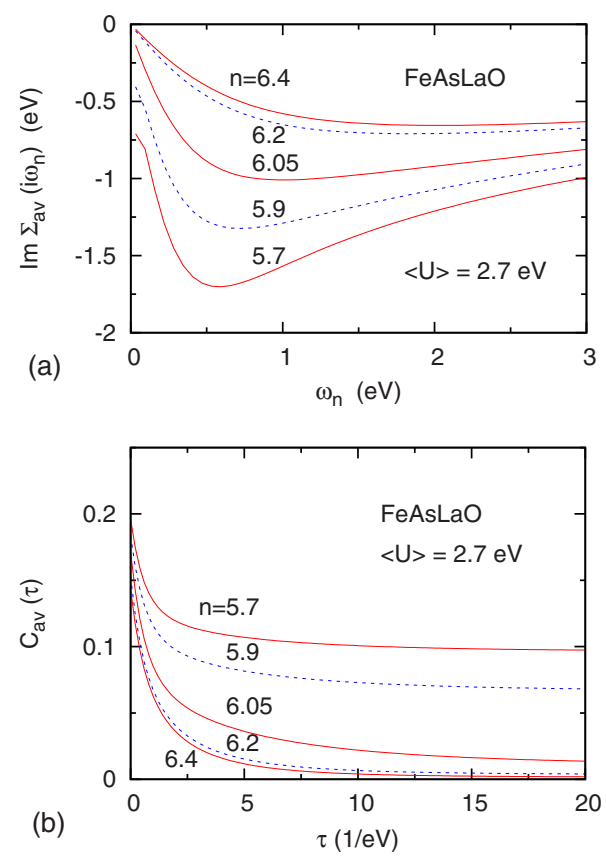

FIG. 5. (Color online) Orbital average of (a) imaginary part of $3 d$ self-energy and (b) spin-spin correlation function for FeAsLaO at various occupancies. The Fermi-liquid to non-Fermi-liquid transition caused by local-moment formation occurs close to $n=6$.

For $n=6$, the low-energy scattering rate $\gamma_{m}=-\operatorname{Im} \Sigma_{m}\left(i \omega_{n}\right.$ $\rightarrow 0)$ and the spin-correlation function reveal non-Fermiliquid behavior associated with the formation of local moments. Fermi-liquid behavior is recovered at electron doping $n>6.2$ whereas hole doping strengthens bad-metallic properties. The important conclusion from this result is that orbital polarization is not a prerequisite for the spin freezing transition. Thus, this picture differs from the orbital-selective, itinerant-localized scenarios proposed in Refs. $28-31$

To understand better to what extent the results for FeSe shown in Fig. 1 depend on the details of the single-particle Hamiltonian, we have carried out analogous ED/DMFT calculations by replacing $H(\mathbf{k})$ with the tight-binding Hamiltonian derived by Graser et al. ${ }^{32}$ for FeAsLaO. Since the $3 d$ density-of-states components of this compound are qualitatively similar to those of FeSe, there ought to be also a spin freezing transition. Indeed, if we retain the FeSe interaction parameters with an average Coulomb energy of $4.2 \mathrm{eV}$, the overall behavior of the low-energy scattering rates and spinspin correlations are qualitatively similar to the ones shown in Fig. 1, except for a less pronounced orbital polarization. For instance, at $n=6, n_{x y}=0.77$ rather than 0.95 . Nevertheless, the Fermi-liquid to non-Fermi-liquid transition also occurs near $n=6.3, \ldots, 6.5$, suggesting that this phenomenon is remarkably robust, i.e., insensitive to the details of the oneelectron properties. These results further support the main point of the degenerate-band example presented in Fig. 4, namely, that the spin freezing transition is not driven by orbital polarization.

According to the work by Miyake et al.,${ }^{14}$ the key difference between FeSe and FeAsLaO is that, because of more 

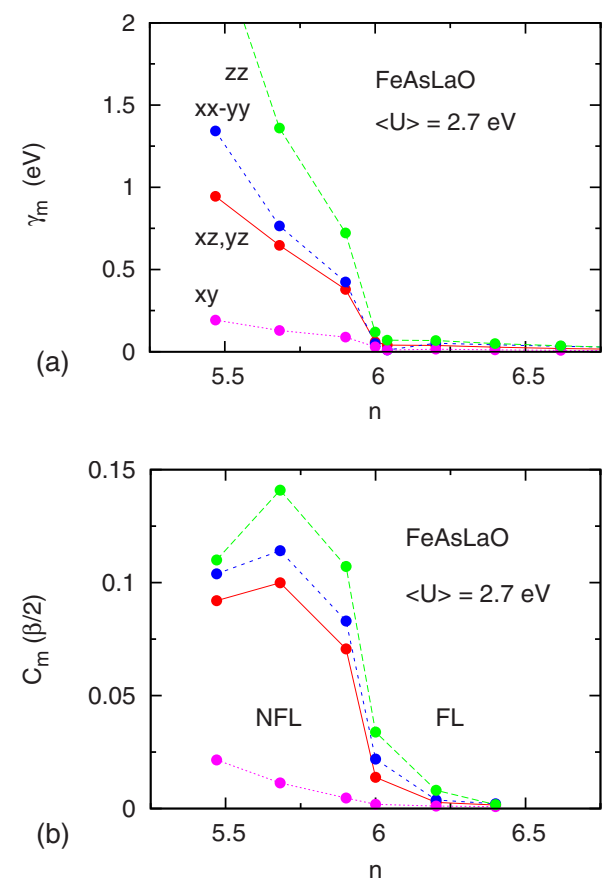

FIG. 6. (Color online) (a) Low-energy scattering rates and (b) spin-spin correlations of FeAsLaO as functions of $3 d$ occupancy. The Fermi-liquid to non-Fermi-liquid transition caused by localmoment formation occurs close to $n=6$. Electron doping maintains Fermi-liquid properties while hole doping leads to bad-metallic behavior.

efficient $d p$ screening and more extended Wannier orbitals, local Coulomb interactions in the latter system are considerably smaller, with $\langle U\rangle \approx 2.7 \mathrm{eV}$ and $\langle J\rangle \approx 0.4 \mathrm{eV}$. In previous ED/DMFT calculations for FeAsLaO (Ref. 19) based on orbital-independent Coulomb and exchange energies we showed that spin freezing may occur at about $U=3 \mathrm{eV}$ for Hund exchange $J=0.75 \mathrm{eV}$. Using the recently published orbital-dependent $U_{m n}$ and $J_{m n}$ matrices derived within constrained RPA, ${ }^{14}$ we are now able to make a more accurate prediction of the spin freezing transition in FeAsLaO. The transfer integrals and interaction parameters are again held fixed at their values for $n=6$. As shown in Fig. 5, the transition now is located almost exactly at $n=6$. Thus, in contrast to FeSe, Fermi-liquid properties in $\mathrm{FeAsLaO}$ prevail and the $3 d$ bands exhibit only moderate effective-mass enhancement, $m^{*} \approx 2, \ldots, 3 .{ }^{16}$ Nonetheless, hole doping $n<6$ gives rise to local-moment formation and bad-metallic behavior, with a finite lifetime at $E_{F}$ while electron doping stabilizes the Fermi-liquid properties. The corresponding orbitaldependent low-energy scattering rates $\gamma_{m}$ and long-time spinspin correlation functions $C_{m}(\beta / 2)$ are given in Fig. 6. Again, the $x y$ orbital is somewhat less correlated than the other $3 d$ components. However, in contrast to FeSe, this anisotropy is less pronounced because to the greater similarity of the FeAsLaO density-of-states components. ${ }^{14}$

On the basis of these results we arrive at the phase diagram depicted in Fig. 7. FeSe is located well inside the nonFermi-liquid phase, whereas FeAsLaO lies on the Fermiliquid side of the spin freezing transition. Both systems

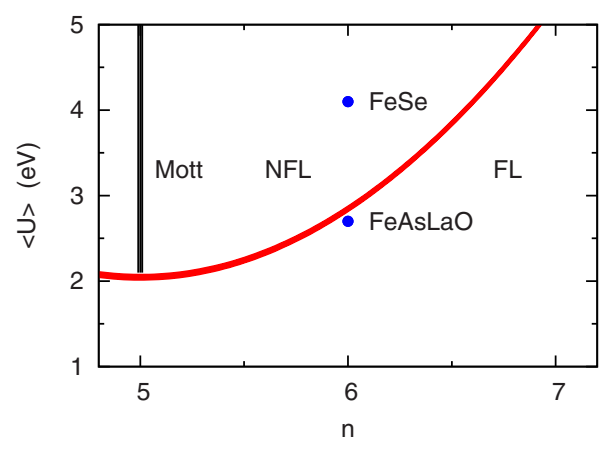

FIG. 7. (Color online) Schematic phase diagram for FeSe and LaFeAsO. Solid curve: spin freezing transition indicating the boundary between Fermi-liquid and non-Fermi-liquid phases. Vertical bar at half filling: Mott insulating phase.

exhibit "parent" Mott phases in the limit $n=5$. In principle, Mott phases might exist also at other integer fillings. In Ref. 19 we have increased $U$ up to $6 \mathrm{eV}(J=0.75 \mathrm{eV})$ without encountering a Mott transition near $n=6$. Thus, away from half filling, a wide bad-metallic region exists well below possible insulating phases at larger $U$. We emphasize that, according to the results shown in Figs. 1, 3, and 4, the onset of non-Fermi-liquid behavior seen in $\operatorname{Im} \Sigma\left(i \omega_{n}\right)$ coincides with the transition of the spin susceptibility from Pauli to Curie-Weiss behavior. The same coincidence was found by Werner et al. ${ }^{17}$

We finally discuss the role of spin-flip and pair-exchange interactions. In Ref. 19 it was shown that the omission of these terms in the many-body impurity treatment leads to a significant shift of the Fermi-liquid to non-Fermi-liquid phase boundary to $\sim 1 \mathrm{eV}$ smaller $\langle U\rangle$. Thus, for FeSe badmetallic behavior would become even stronger. Since FeAsLaO, however, lies very close to the phase boundary, this approximation has severe consequences. Instead of a Fermi-liquid characterized by moderate effective-mass enhancement, this compound is then also bad metallic, in conflict with experiments.

The present work is based on a consistent combination of single-particle Hamiltonian, constrained RPA interaction parameters, and DMFT many-electron calculations within a $5 \times 5 d$ electron basis. It would be interesting to compare the spin freezing transition obtained within this scheme to analogous formulations within a $p d$ electron basis, such as the one used in Ref. 21

\section{CONCLUSION}

In summary, we have evaluated the effect of local correlations in FeSe, using accurate single-particle properties and constrained RPA results for the orbital-dependent Coulomb and exchange matrices, combined with ED/DMFT. The finite scattering rates derived from the $3 d$ components of the selfenergy are shown to be linked to the formation of local moments. Thus, FeSe is bad metallic, in agreement with experimental findings. Spin freezing ceases for large electron doping, giving rise to Fermi-liquid behavior. In contrast, hole doping enhances bad metallicity. Applying the same scheme 
to FeAsLaO we find that, as a result of the more efficiently screened Coulomb interactions, local-moment formation is confined to hole doping $n<6$. Thus, FeAsLaO and FeSe seem to be located on opposite sides of the Fermi-liquid to non-Fermi-liquid spin freezing transition.

\section{ACKNOWLEDGMENTS}

A.L. likes to thank M. Aichhorn, R. Arita, E. Gull, K. Haule, A. Millis, and Ph. Werner, for fruitful discussions, and the KITP Santa Barbara for hospitality. The calculations were carried out on the Jülich Juropa computer.
${ }^{1}$ Y. Kamihara, T. Watanabe, M. Hirano, and H. Hosono, J. Am. Chem. Soc. 130, 3296 (2008).

${ }^{2}$ F. C. Hsu, J.-Y. Luo, K.-W. Yeh, T.-K. Chen, T.-W. Huang, Ph. M. Wu, Y.-C. Lee, Y.-L. Huang, Y.-Y. Chu, D.-C. Yan, and M.-K. Wu, Proc. Natl. Acad. Sci. U.S.A. 105, 14262 (2008).

${ }^{3}$ W. Malaeb, T. Yoshida, T. Kataoka, A. Fujimori, M. Kubota, K. Ono, H. Usui, K. Kuroki, R. Arita, H. Aoki, Y. Kamihara, M. Hirano, and H. Hosono, J. Phys. Soc. Jpn. 77, 093714 (2008).

${ }^{4}$ R. Yoshida, T. Wakita, H. Okazaki, Y. Mizuguchi, S. Tsuda, Y. Takano, H. Takeya, K. Hirata, T. Muro, M. Okawa, K. Ishizaka, S. Shin, H. Harima, M. Hirai, Y. Muraoka, and T. Yokoya, J. Phys. Soc. Jpn. 78, 034708 (2009).

${ }^{5}$ A. Yamasaki, S. Imada, K. Takase, T. Muro, Y. Kato, H. Kobori, A. Sugimura, N. Umeyama, H. Sato, Y. Hara, N. Miyakawa, and S. Ikeda, arXiv:0902.3314 (unpublished).

${ }^{6}$ Y. Xia, D. Qian, L. Wray, D. Hsieh, G. F. Chen, J. L. Luo, N. L. Wang, and M. Z. Hasan, Phys. Rev. Lett. 103, 037002 (2009).

${ }^{7}$ K. Nakayama, T. Sato, P. Richard, T. Kawahara, Y. Sekiba, T. Qian, G. Chen, J. Luo, N. Wang, H. Ding, and T. Takahashi, arXiv:0907.0763 (unpublished).

${ }^{8}$ A. Tamai, A. Y. Ganin, E. Rozbicki, J. Bacsa, W. Meevasana, P. D. C. King, M. Caffio, R. Schaub, S. Margadonna, K. Prassides, M. J. Rosseinsky, and F. Baumberger, Phys. Rev. Lett. 104, 097002 (2010).

${ }^{9}$ G. F. Chen, Z. G. Chen, J. Dong, W. Z. Hu, G. Li, X. D. Zhang, P. Zheng, J. L. Luo, and N. L. Wang, Phys. Rev. B 79, 140509(R) (2009).

${ }^{10}$ I. Pallecchi, G. Lamura, M. Tropeano, M. Putti, R. Viennois, E. Giannini, and D. Van der Marel, Phys. Rev. B 80, 214511 (2009).

${ }^{11}$ M. Tropeano, I. Pallecchi, M. R. Cimberle, C. Ferdeghini, G. Lamura, M. Vignolo, A. Martinelli, A. Palenzona, and M. Putti, Supercond. Sci. Technol. 23, 054001 (2010).

${ }^{12}$ Y. Song, J. Hong, B. Min, K. Lee, M. Jung, J. Rhyee, and Y. Kwon, arXiv:0911.2045 (unpublished).

${ }^{13}$ B. C. Sales, A. S. Sefat, M. A. McGuire, R. Y. Jin, D. Mandrus, and Y. Mozharivskyj, Phys. Rev. B 79, 094521 (2009).

${ }^{14}$ T. Miyake, K. Nakamura, R. Arita, and M. Imada, J. Phys. Soc. Jpn. 79, 044705 (2010).

${ }^{15}$ A. Georges, G. Kotliar, W. Krauth, and M. J. Rozenberg, Rev. Mod. Phys. 68, 13 (1996).

${ }^{16}$ For Ising exchange similar results were found by M. Aichhorn, L. Pourovskii, V. Vildosola, M. Ferrero, O. Parcollet, T. Miyake, A. Georges, and S. Biermann, Phys. Rev. B 80, 085101 (2009).

${ }^{17}$ P. Werner, E. Gull, M. Troyer, and A. J. Millis, Phys. Rev. Lett. 101, 166405 (2008).

${ }^{18}$ K. Haule, J. H. Shim, and G. Kotliar, Phys. Rev. Lett. 100, 226402 (2008); K. Haule and G. Kotliar, New J. Phys. 11, 025021 (2009).

${ }^{19}$ H. Ishida and A. Liebsch, Phys. Rev. B 81, 054513 (2010).

${ }^{20}$ L. Craco, M. Laad, and S. Leoni, arXiv:0910.3828 (unpublished).

${ }^{21}$ M. Aichhorn, S. Biermann, T. Miyake, A. Georges, and M. Imada, Phys. Rev. B 82, 064504 (2010).

${ }^{22}$ P. Hansmann, R. Arita, A. Toschi, S. Sakai, G. Sangiovanni, and K. Held, Phys. Rev. Lett. 104, 197002 (2010).

${ }^{23}$ M. Caffarel and W. Krauth, Phys. Rev. Lett. 72, 1545 (1994).

${ }^{24}$ A. Subedi, L. Zhang, D. J. Singh, and M. H. Du, Phys. Rev. B 78, 134514 (2008).

${ }^{25}$ K.-W. Lee, V. Pardo, and W. E. Pickett, Phys. Rev. B 78, 174502 (2008).

${ }^{26}$ I. Souza, N. Marzari, and D. Vanderbilt, Phys. Rev. B 65, 035109 (2001)

${ }^{27}$ F. Aryasetiawan, M. Imada, A. Georges, G. Kotliar, S. Biermann, and A. I. Lichtenstein, Phys. Rev. B 70, 195104 (2004).

${ }^{28}$ J. Wu, Ph. Phillips, and A. H. Castro Neto, Phys. Rev. Lett. 101, 126401 (2008)

${ }^{29}$ A. Hackl and M. Vojta, New J. Phys. 11, 055064 (2009).

${ }^{30}$ S.-P. Kou, T. Li, and Z.-Y. Weng, EPL 88, 17010 (2009).

${ }^{31}$ L. de' Medici, S. R. Hassan, and M. Capone, J. Supercond. Novel Magn. 22, 535 (2009).

${ }^{32}$ S. Graser, T. A. Maier, P. J. Hirschfeld, and D. J. Scalapino, New J. Phys. 11, 025016 (2009). 